\title{
Evolutionary approach reveals impact of missense variants in autism
}

\author{
BY CHLOE WILLIAMS
}

7 JULY 2021

Leveraging cross-species data on the evolution of proteins can help make sense of subtle genetic variants in people with autism and identify hundreds of new genes that may contribute to the condition, according to a new analysis.

The work focuses on 'missense' variants, which alter a single amino acid in a protein and often have mild effects. Although researchers have identified thousands of missense variants in people with autism, parsing out those that contribute to the condition has been challenging.

"The impact of a mutation that changes one amino acid into another in a protein is difficult to interpret," says Olivier Lichtarge, professor of molecular and human genetics at Baylor College of Medicine in Houston, who led the recent research. It might alter how the protein folds, degrades, gets transported or interacts with other molecules, and predicting that outcome is complex, he says.

To identify missense variants linked to autism, Lichtarge and his colleagues used an approach known as 'evolutionary action,' which involves comparing a protein's amino acid sequences across different species to infer a missense variant's likely impact.

Using this strategy, the team identified missense variants in 398 genes that might contribute to autism. Some are known autism-linked genes, such as RELN, PTEN and SYNGAP1, but others have not previously been tied to the condition.

The approach "seems to indeed identify important mutations in the context of autism," says Ivan lossifov, associate professor at Cold Spring Harbor Laboratory in New York, who was not involved in the work. Although researchers have developed many ways to assess the impact of missense variants, spotting those linked to autism is still an important problem in the field, he says, and "this seems to be a good way of approaching it." 


\section{Evolutionary experiment:}

Lichtarge and his colleagues searched for non-inherited, or de novo, missense variants in 2,384 people with autism and 1,792 of their unaffected siblings. The data came from the Simons Simplex Collection, a repository of genetic and trait information from families with one autistic child. (The dataset is funded by the Simons Foundation, Spectrum's parent organization.)

The autistic participants have 1,418 de novo missense variants that affect 1,269 genes, and their siblings have 976 missense variants that affect 911 genes, the researchers reported in May in Science Translational Medicine.

The team calculated each variant's evolutionary action score on a scale from 0 to 100; the higher the score, the more likely a variant is to damage the gene's corresponding protein. The score considers two factors: the sensitivity of a particular spot in a protein's amino acid sequence to variants and the severity of the disruption caused by an amino acid change.

To measure sensitivity, the team used existing databases to compare amino acid sequences in proteins across various species for each mutated gene's associated protein. They then gauged the evolutionary distance linked to a change at a specific position in the sequence. If an alteration was associated with a large evolutionary distance, variants at that site were considered likely to alter the function of the corresponding protein.

To assess the severity of a disruption caused by an amino acid change, the team measured how often a particular amino acid is swapped for another in any protein across species. A change that occurred rarely throughout evolution suggested that the new amino acid has different properties than the one it replaced and the alteration may have been damaging.

Although people with autism have more de novo missense variants than their siblings, the distribution of scores in the two groups was not significantly different, and the researchers say that without relying on existing knowledge about autism-linked genes, they would have been unable to identify which of the affected genes contribute to the condition.

So the team grouped variants based on 368 biological pathways - focusing on the variants in the 1,792 autistic people who have matched siblings - and examined the distribution of evolutionary action scores. Only 23 pathways were biased toward high-impact variants, many of which are tied to neurodevelopment, neuronal signaling and the development of neuronal projections called axons.

The high-impact pathways include 398 genes, many of which appear in the SFARI Gene database of autism-linked genes. (SFARI Gene is funded by the Simons Foundation.) Of those 398 genes, 28 were not categorized as "high confidence" in 2017 but were listed as such in 2020. 


\section{Spectrum | Autism Research News}

https://www.spectrumnews.org

These findings suggest that the evolutionary action approach could help identify candidate genes for future research, the researchers say.

\section{Severity score:}

In a further analysis, the team split people with autism into three groups based on their intelligence quotients (IQs). For each person, they tallied the evolutionary action score of variants within the 398 genes.

People with the lowest IQs have the highest-impact variants in the prioritized genes, supporting a link between the variants and autism, the researchers say. Summed scores of rare, inherited missense variants also track with $I Q$, another test shows.

Previous studies have not found a statistically significant link between rare, inherited missense variants and the severity of autism traits, says Yufeng Shen, associate professor of systems biology and biomedical informatics at Columbia University, who was not involved in the research. So the evolutionary approach may be useful for uncovering the role these variants play in the condition, he says.

One limitation of the study is that the researchers did not systematically compare the evolutionary action approach with other methods commonly used to identify harmful missense variants, Shen says. "Without a comparison to other methods, it's very hard to assess how much of a contribution this method has to autism research."

The researchers also caution that IQ is only one measure of the severity of autism traits and that a person's impact score is not necessarily predictive of their IQ.

The findings are just the "tip of the iceberg," Lichtarge says. As researchers analyze more data, they may eventually be able to use evolutionary action scores to find out how a person's variants affect their genetic traits in a more individualized way.

Cite this article: https://doi.org/10.53053/VESX6574 